\title{
Effects of in situ climate warming on monarch caterpillar (Danaus plexippus) development
}

Nathan P Lemoine, Jillian N Capdevielle, John D Parker

Climate warming will fundamentally alter basic life history strategies of many ectothermic insects. In the lab, rising temperatures increase growth rates of lepidopteran larvae, but also reduce final pupal mass and increase mortality. Using in situ field warming experiments on their natural host plants, we assessed the impact of climate warming on development of monarch (Danaus plexippus) larvae. Monarchs were reared on Asclepias tuberosa grown under 'Ambient' and 'Warmed' conditions. We quantified time to pupation, final pupal mass, and survivorship. Warming significantly decreased time to pupation, such that an increase of $1^{\circ} \mathrm{C}$ corresponded to a 0.5 day decrease in pupation time. In contrast, survivorship and pupal mass were not affected by warming. Our results indicate that climate warming will speed the developmental rate of monarchs, influencing their ecological and evolutionary dynamics. However, the effects of climate warming on larval development in other monarch populations and at different times of year should be investigated. 
1 Effects of in situ warming on monarch caterpillar (Danaus plexippus) development

2

3 Nathan P. Lemoine ${ }^{1,4}$, Jillian Capdevielle 2 , John D. Parker ${ }^{3}$

4

$5 \quad{ }^{1}$ Florida International University

6 MSB 350

7 Department of Biological Sciences

8 North Miami, Florida 33181

${ }^{2}$ University of California - Berkeley

Department of Integrative Biology

Berkeley, CA 94720

${ }^{3}$ Smithsonian Environmental Research Center

647 Contees Wharf Rd.

Edgewater, MD 21037

${ }^{4}$ corresponding author:

Nathan P. Lemoine

Florida International University - MSB 350

North Miami, FL 33181

email:nlemo001@fiu.edu

phone: 305-919-4109

Running Header: Lemoine et al: Warming effects on monarch development 


\section{Abstract}

Climate warming will fundamentally alter the development of many ectothermic insects; understanding how elevated temperatures affect insect development is becoming increasingly important. Yet most studies examining the relationship between temperature and insect development do so in highly controlled laboratory settings. While useful, laboratory studies cannot incorporate natural variability in temperatures or behavioral thermoregulation by insects. Using in situ field warming experiments, we assessed the impact of experimental warming on development of monarch butterfly larvae (Danaus plexippus). Monarch larvae were reared on butterfly weed (Asclepias tuberosa) grown under 'Ambient' and 'Warmed' conditions. We quantified time to pupation, final pupal mass, and survivorship. Monarch larval development proceeded more rapidly at higher temperatures, and this effect was even greater for monarch larvae reared under 'Warmed' conditions. In contrast, survivorship and pupal mass were not affected by warming. More rapid developmental rates of monarch larvae will alter their ecological and evolutionary dynamics by potentially speeding up northward migrations and increasing the number of generations during the summer breeding season. Our results contrast

41 those from laboratory experiments, wherein rising temperatures increase growth rates of lepidopteran larvae, but also reduce final pupal mass and increase mortality. Therefore, more studies assessing the impact of warming and climate change on monarch biology are needed. 


\section{Introduction}

Modified temperature regimes caused by climate change will fundamentally alter insect life cycles. As with other insects, lepidopteran larval development is temperature-dependent. Warming increases growth rates and survivorship; however both growth and survival decline rapidly once temperatures exceed an individual's thermal optimum (Kingsolver et al. 2006, Kingsolver and Woods 1997). The effects of elevated temperatures on lepidopteran larval development have, to date, been mostly examined in highly controlled lab settings. Such laboratory experiments cannot incorporate natural temperature fluctuations that affect larval development and survival (Zalucki 1982) or changes in insect behavior (i.e. behavioral thermoregulation, predator avoidance). Furthermore, warming alters plant nutritional quality (Veteli et al. 2002), and lab experiments often use artificial foods (Kingsolver et al. 2006, Lee and Roh 2010) or leaf material that was not grown under elevated temperatures (Lemoine et al. 2014). Extrapolating results from laboratory experiments to natural settings is therefore problematic. Field experiments are necessary to identify how elevated temperatures influence insect development in a more natural, albeit, variable environment.

Monarch butterflies (Danaus plexippus) are a charismatic species found throughout North America and are well known for their annual migrations between Mexico and northern United States and southern Canada. Monarch migrations have been extensively studied, focusing on factors that influence migration success and population size (Reppert et al. 2010, Flockhart et al. 2015), potential overwintering and migratory habitat loss (Oberhauser and Peterson 2003, Pleasants and Oberhauser 2012, Sáenz-Romero et al. 2012), and overwintering behavior (Masters et al. 1988). Reductions in overwintering and migratory habitat caused by changes in climate and land-use have stimulated research on thermal constraints experienced by migratory 
67 adults and larvae, the need for cool night time temperatures to induce reproductive diapause in

68 adult monarchs (Goehring and Oberhauser 2002, Guerra and Reppert 2013), and the threat posed

69 by spring droughts that reduce monarch population sizes in their summer breeding grounds

70 (Zipkin et al. 2012).

As with all insect species, monarch larval growth, consumption, and mortality rates depend upon environmental temperatures (Zalucki 1982, Goehring and Oberhauser 2002, York and Oberhauser 2002, Lemoine et al. 2014). Prolonged exposure to extreme heat reduces larval growth and survival rates in laboratory experiments (Zalucki 1982, York and Oberhauser 2002). Although warming alters the nutritional quality of monarchs' milkweed host plants (Couture et al. 2015), few studies consider concurrent effects of warming on both monarch and milkweed (but see Couture et al. 2015). Milkweed nitrogen, lignin, and fiber content increase under elevated temperatures (Couture et al. 2015). Given that elevated temperatures affect both monarchs and milkweeds simultaneously, the relationship between temperature and monarch larval development and survival might be fundamentally different under climate warming. Field experiments are necessary to explore how warming affects monarch larval development in a scenario that incorporates natural temperature variability and changes in host plant quality. Here, we report results from an in situ warming experiment designed to assess how variable field conditions. We hypothesized that development time would decrease with rising temperatures under ambient conditions, as has commonly been reported for monarch larvae (Zalucki 1982). However, this relationship between development time and temperature would be significantly stronger under warming since milkweed grown under elevated temperatures contains significantly more nitrogen (Couture et al. 2015). We expected that pupal mass and 
90

survival would decrease with rising temperatures (Zalucki 1982, York and Oberhauser 2002), but that warming would weaken these effects due to the effects of elevated temperatures on milkweed nutritional quality (Couture et al. 2015).

\section{Methods}

All experiments were conducted at the Smithsonian Environmental Research Center in Edgewater, MD. The experiment consisted of 16 replicate $2 \times 2 \mathrm{~m}$ garden beds. Garden beds were assigned to temperature treatments in a completely randomized design. Warming treatments were imposed using a single Kalglo MRM-1215 1500W (Kalglo Electronics Company, Bethlehem, PA) heater installed $1.5 \mathrm{~m}$ from the ground over half of the garden beds. An aluminum frame of the same shape and size as the heaters was hung over the remaining garden beds to mimic any shading effects ( $n=8$ garden beds per temperature treatment). In each garden bed, $1 \mathrm{~m}$ long, $20 \mathrm{~cm}$ high aluminum sheets were driven $10 \mathrm{~cm}$ into the soil to quarter the $2 \times 2$ m garden bed into four $1 \times 1$ m subplots. In the fall of 2013, butterfly weed Asclepias tuberosa was sown into two of the subplots within each garden bed, resulting in a density of $\sim 4$ plants per subplot (the remaining two subplots were used for other experiments). We chose $A$. tuberosa over the $A$. syriaca because $A$. syriaca can grow to $>2 \mathrm{~m}$ tall, surpassing the height of our heaters. The experimental unit was therefore $321 \times 1 \mathrm{~m}$ subplots ( $n=16$ per temperature) within the sixteen garden beds.

We placed Onset HOBO temperature loggers in the center of each garden bed to record air temperature in 10-minute intervals over the course of the experiment. In 'Ambient' treatments, average daytime temperatures were $25.2 \pm 1.4^{\circ} \mathrm{C}$ and average nighttime temperatures of $19.9 \pm 2.0^{\circ} \mathrm{C}$. Maximum daytime temperatures at our study site were $30.7 \pm 2.5^{\circ} \mathrm{C}$, while 
113 minimum nighttime temperatures were $18.2 \pm 2.3^{\circ} \mathrm{C}$. Since air temperature measurements may

114 not accurately reflect the heating achieved by infrared heaters (Kimball et al. 2008), we verified

115 heating treatments using a handheld IR thermometer to measure temperatures on a plastic sphere

116 mounted $0.5 \mathrm{~m}$ from the ground placed in the middle each experimental subplot at midnight.

117 Nighttime IR gun measurements verified that heaters raised surface temperatures by $\sim 4^{\circ} \mathrm{C}$ on

118 average $(p<0.001)$, which is below severe projections of a $6^{\circ} \mathrm{C}$ increase in temperature but

119 above the more conservative estimate of a $2^{\circ} \mathrm{C}$ temperature increase by 2100 (IPCC 2007).

120 In August 2014, monarch eggs and larvae were gathered from $A$. syriaca within nearby

121 old-growth fields. Eggs and larvae were reared in mesh cages and fed fresh $A$. syriaca leaves

122 daily until they reached the third instar. Larval development was checked continuously

123 throughout the day. First or second instars escaped the mesh bags easily and thus were not used.

124 Immediately after molting to the third instar, larvae were randomly assigned to a temperature

125 treatment ('Ambient', 'Warmed') and a single larva was placed on a single $A$. tuberosa within a

126 randomly chosen plot ( $n=15, n=18$ for 'Ambient' and 'Warmed' treatments, respectively). A

$12720 \times 30.5 \mathrm{~cm}$ organza mesh bag was placed over the plant to retain the monarch. If the monarch

128 larva consumed the entire host plant, they were transferred to another plant within the same

129 subplot. Time to pupation was recorded as the number of hours between experiment initiation

130 and onset of chrysalis formation, and this number was converted to number of days

131 (development hour / 24). Dead individuals were recorded and removed from the host plant.

132 Chrysalids were transported to the lab and weighed to obtain final pupal mass.

133 We measured three plant traits (specific leaf area (SLA), water content, and latex

134 production) to determine whether warming effects on monarch development might be mediated

135 through warming effects on plant traits. At the end of the experiment, two newly expanded 
136 leaves were collected from each plant. For one leaf, we measured leaf area, obtained a fresh wet

137 mass, and then dried the leaf to obtain a dry mass. We calculated specific leaf area (SLA) as area

138 / dry mass and percent water content as $(1-$ dry mass $(\mathrm{g}) /$ fresh mass $(\mathrm{g})) * 100$. Using the second

139 leaf, we determined latex production by cutting the tip of the leaf and blotting all latex onto a

140 dry, pre-weighed piece of filter paper (Agrawal 2005). The filter paper was dried again and latex

141 concentration calculated as the difference in post- and pre-latex filter weights divided by leaf

142 area (Agrawal 2005).

143 Although heaters raised temperatures of 'Warmed' plots by $\sim 4^{\circ} \mathrm{C}$ on average, plots

144 varied considerably in temperature due to different light levels across the experimental garden

145 and varying plant biomass within each plot. We therefore measured temperature with a handheld

146 infrared thermometer in each subplot during the night at the end of the experiment. For

147 consistency, we recorded temperature of a white plastic sphere mounted $0.5 \mathrm{~m}$ from the ground

148 in the middle of each subplot. We then treated temperature as a quantitative rather than

149 categorical variable in all analyses. Note that these measures reflect relative differences in

150 temperature among plots that should be relatively constant over the experiment.

151 We used an ANCOVA design to regress days ${ }^{-1}$ until pupation and final pupal mass

152 against night-time temperatures as measured by the IR gun. We included temperature treatment

153 as a covariate, which allows for the possibility that slopes differ between temperature treatments.

154 Mortality was assessed using logistic regression that also included night-time temperature and its

155 interaction with temperature treatment, as in the ANCOVA. Although monarchs experience

156 mortality as pupae, brief exposure to prolonged temperatures did not alter pupal mortality rates

157 and third instars were the most sensitive to temperature increases (York and Oberhauser 2002).

158 Thus, our experiment likely captured most of the influence of temperature on larval survival. 

were conducted using Python v2.7 with the 'numpy', 'pandas', and 'statsmodels' modules

161 (McKinney 2010, Seabold and Perktold 2010, Walt et al. 2011).

\section{Results}

Time to pupation decreased with increasing temperature, but did so differently under

'Ambient' and 'Warmed' conditions (interaction: $p<0.041$ ) (Fig. 1). At the lowest temperature in 'Ambient' treatments, $12.6^{\circ} \mathrm{C}$, monarch larvae required 12.2 days to transition between third instar and pupa. At the warmest temperature achieved in the 'Warmed' plots, monarch larvae required only 10.0 days to pupate. Importantly, the relationship between temperature and time to development varied among treatments (Fig. 1). When reared under 'Ambient' conditions, larval development time decreased by $\sim 0.4$ days per $1^{\circ} \mathrm{C}$ increase in temperature. In 'Warmed' plots, larval development time decreased by $\sim 0.7$ days per $1^{\circ} \mathrm{C}$ increase in temperature. Climate change may therefore speed larval development by $\sim 0.7-2.4$ days, depending on the severity of temperature increases.

Air temperature measurements do not accurately reflect the intensity of infrared heating because infrared energy warms surfaces and not the air (Kimball et al. 2008), calculations of degree-days may not accurately reflect the underlying temperature treatments. Still, we calculated the number of degree days experienced by each individual for which there was adequate temperature data following the simple averaging method, since temperatures remained within the upper and lower thermal limits throughout the experiment (Allen 1976). Monarch caterpillars experienced $\sim 155 \pm 17$ degree days, and this did not differ between temperature 
181 treatments $(p=0.978)$. Thus, monarch larvae accumulated their required number of degree days

182 faster in the warming treatment than in the ambient treatment.

183 Temperature had no effect on pupal mass $\left(p=0.454, R^{2}=0.023\right)$. Similarly, mortality

184 was low throughout the experiment (18\%) and independent of temperature $\left(p=0.610\right.$, pseudo- $R^{2}$

$185=0.01)$.

186 Warming had no effect on any measured plant trait. SLA $\left(p=0.940, R^{2}=0\right)$, percentage

187 water content $\left(p=0.313, R^{2}=0.05\right)$, and latex concentration $\left(p=0.739, R^{2}=0.01\right)$ all did not

188 vary with temperature. Thus, any effects of warming on monarch development time were direct

189 effects of temperature on monarch physiology rather than being mediated through the plant traits

190 we measured.

191

192

\section{Discussion}

Our study indicates that warming accelerates monarch larval development but has little

194 effect on larval mortality or pupal mass at our study site. This is consistent with numerous studies showing positive correlations between larval development and temperature (Kingsolver and Woods 1997, Bale et al. 2002). Since warming increases larval growth rates, lepidopteran larvae reach critical mass needed for pupation earlier and proceed through larval stadia more quickly. Monarch larvae developed more rapidly from the third instar but experienced roughly the same number of degree days. Our results suggest that climate warming might facilitate monarch larval development through later instars under moderate climate change scenarios at sites with relatively cool temperatures, potentially increasing the number of generations in the temperate summer breeding grounds of eastern migratory monarch populations. 

temperatures on monarch caterpillar development and survival. Short-term, extreme heat stress can have weak negative effects on pupal mass (York and Oberhauser 2002). Likewise, constant temperatures above $28^{\circ} \mathrm{C}$ induced high mortality rates in monarch larvae (Zalucki 1982, York and Oberhauser 2002). However, these studies used either pulses of extremely high temperatures (i.e. $36^{\circ} \mathrm{C}$ ) or held monarch larvae at a constant temperature (i.e. $\left.28^{\circ} \mathrm{C}\right)$. Ambient, maximum daytime temperatures averaged $30{ }^{\circ} \mathrm{C}$ during our experiment; warming increased this maximum to $32-34^{\circ} \mathrm{C}$. Although these temperatures are above the thermal optimum of monarch survival, we found no effect of in situ warming on either pupal mass or survival of older monarch larvae. Eggs and first instar larvae are resistant to high temperatures, with third instars, fourth instars, and pupae being the most sensitive to extreme heat (Zalucki et al. 1982, York and Oberhauser 2002). As temperatures exceeded $28^{\circ} \mathrm{C}$ for less than $20 \%$ of the full 24 hour day, it is likely that diel and daily temperature fluctuations mitigated the lethality of high temperatures. during their northward migration: maximum March temperatures in Texas average $23.5 \pm 2.4^{\circ} \mathrm{C}$, maximum April temperatures in Iowa and the midwestern US average $20.7 \pm 1.5^{\circ} \mathrm{C}$, and maximum May temperatures in the Great Lakes region average $18 \pm 2.3^{\circ} \mathrm{C}$ (averages based on 50 year weather station data provided by WorldClim). Even maximum temperatures during the summer breeding season in the Great Lakes region are typically lower than at our study site, averaging $26.0 \pm 2.3^{\circ} \mathrm{C}$ compared to $30.7 \pm 2.5^{\circ} \mathrm{C}$ at during our experiment. We found no influence of increased temperatures on larval monarch pupal mass and survival at our study site, which had temperatures well above those in other important breeding ranges. Indeed, 
226 temperatures in these ranges rarely exceed the thermal optimum of $28^{\circ} \mathrm{C}$ and do not exceed the

227 critical thermal maximum of $36^{\circ} \mathrm{C}$ (Zalucki 1982, York and Oberhauser 2002). Thus, climate

228 change is unlikely to raise temperatures to a range that is detrimental to monarch larval

229 performance.

230 Though monarch larval development proceeded more rapidly when exposed to high

231 temperatures, this effect was stronger on plants grown under warmed conditions. Increased

232 sensitivity to temperature in 'Warmed' plots likely stems from altered plant nutritional content.

233 Though we found no different in A. tuberosa leaf characteristics between 'Ambient' and

234 'Warmed' treatments, elevated temperatures alter foliar water content, nutritional content, and

235 secondary metabolite concentrations of numerous plant species (Zvereva and Kozlov 2006). In

236 particular, milkweed nitrogen content increases at elevated temperatures (Couture et al. 2015).

237 Insect development proceeds more rapidly at high temperatures on nitrogen-rich plants (Lemoine

238 et al. 2013, Lemoine et al. 2014). It is therefore likely that increased foliar nitrogen content of $A$.

239 tuberosa grown under elevated temperatures is responsible for the greater sensitivity of monarch

240 larvae to rising temperatures.

241 Our paper demonstrates that climate warming may minimally impact the development of

242 monarch larvae in temperate regions. Though numerous laboratory studies have reported

243 detrimental impacts of extreme temperatures on monarch larval development and survival

244 (Zalucki 1982, York and Oberhauser 2002), our field experiment demonstrated that in situ

245 warming had little influence on larval survival or pupal mass even in a site with extreme daytime

246 temperatures. Rising temperatures may, however, have other important effects on monarch

247 larvae. Monarch larvae may, for example, suffer higher parasitism rates at high temperatures as

248 occurs in other insect-parasitoid pairs (Bezemer et al. 1998). Predatory insects also increase their 
249 attack and ingestion rates at high temperatures, suggesting that predation pressure on monarch

250 larvae may increase substantially under warming (Rall et al. 2010). Furthermore, landscape level

251 distributions of milkweed host plants may be substantially different at elevated temperatures.

252 Warming may reduce the availability of Asclepias host plants during the northward migration via

253 increased drought or drastically alter the geographic range of Asclepias host plants (Zipkin et al.

254 2012, Lemoine 2015). Thus, this research establishes an important baseline for future work

255 considering numerous other consequences of increased temperature on monarch larval

256 performance and survival. 


\section{Acknowledgements}

258 We thank S. Cook-Patton, D. Doublet, and M. Palmer for their assistance during this project. We

259 thank L. Higley, J. Pleasants, R. Peterson, and one anonymous reviewer for their helpful

260 comments and suggestions. 


\section{References}

262

263

264

265

266

267

268

269

270

271

272

273

274

275

276

277

278

279

280

281

282

283

284

285

286

287

288

289

290

291

292

293

294

295

296

297

298

299

300

301

Agrawal, A. 2005. Natural selection on common milkweed (Asclepias syriaca) by a community of specialized herbivores. Evol. Ecol. Res. 7: 651-667.

Allen, J. C. 2006. A modified sine wave method for calculating degree days. Env. Entomol. 5: 88-396.

Bale, J. S., G. J. Masters, I. D. Hodkinson, C. Awmack, T. M. Bezemer, V. K. Brown, J. Butterfield, A. Buse, J. C. Coulson, J. Farrar, J. E. G. Good, R. Harrington, S. Hartley, T. H. Jones, R. L. Lindroth, M. C. Press, I. Symrnioudis, A. D. Watt, J. B. Whittaker. 2002. Herbivory in global climate change research: direct effects of rising temperature on insect herbivores. Glob. Change Bio. 8:1-16.

Bezemer, T. M., T. H. Jones, and K. J. Knight. 1998. Long-term effects of elevated $\mathrm{CO}_{2}$ and temperature on populations of the peach potato aphid Myzus persicae and its parasitoid Aphidius matricariae. Oecologia 116: 128-135.

Couture, J. J., S. P. Serbin, and P. A. Townsend. 2015. Elevated temperature and periodic water stress alter growth and quality of common milkweed (Asclepias syriaca) and monarch (Danaus plexippus) larval performance. Arthropod-Plant Inte. 9: 149-161.

Flockhart, D. T. T., J.-B. Pichancourt, D. R. Norris, and T. G. Martin. 2015. Unravelling the annual cycle in a migratory animal: breeding-season habitat loss drives population declines of monarch butterflies. J. Anim. Ecol. doi:10.1111/1365-2656.12253.

Goehring, L. and K. S. Oberhauser. 2002. Effects of photoperiod, temperature, and host plant age on induction of reproductive diapause and development time in Danaus plexippus. Ecol. Entomol. 27: 674-685.

Guerra, P. A. and S. M. Reppert. 2013. Coldness triggers northward flight in remigrant monarch butterflies. Curr. Biol. 23: 419-423.

IPCC. 2007. Climate change 2007: synthesis report.

Kimball, B. A., M. M. Conley, S. Wang, X. Lin, C. Luo, J. Morgan, and D. Smith. 2008. Infrared heater arrays for warming ecosystem field plots. Global Change Biol. 14: 309320.

Kingsolver, J. G., G. Schlichta, G. J. Ragland, and K. R. Massie. 2006. Thermal reaction norms for caterpillar growth depend on diet. Evol. Ecol. Res. 8: 705-715. 
Kingsolver, J. G. and H. A. Woods. 1997. Thermal sensitivity of growth and feeding in Manduca sexta caterpillars. Physiol. Zool. 70: 631-638.

Lee, K. P. and C. Roh. 2010. Temperature-by-nutrient interactions affecting growth rate in an insect ectotherm. Entomol. Exp. Appl. 136: 151-163.

Lemoine, N. P., W. A. Drews, D. E. Burkepile, and J. D. Parker. 2013. Increased temperature alters feeding behavior of a generalist herbivore. Oikos 122: 1669-1678.

Lemoine, N. P., D. E. Burkepile, and J. D. Parker. 2014. Variable effects of temperature on insect herbivory. PeerJ 2: e376.

Lemoine, N. P. 2015. Climate change may alter monarch spring migration and summer breeding ground distributions. Plos One 10:e0118614

Masters, A. R., S. B. Malcolm, and L. P. Brower. 1988. Monarch butterfly (Danaus plexippus) thermoregulatory behavior and adaptations for overwintering in Mexico. Ecology 69: 458-467.

McKinney, W. 2010. Data structures for statistical computing in Python, pp. 51-56. In Proceedings, 9th Python in Science Conference, June 28 - July 3, Austin, TX.

Oberhauser, K. and A. T. Peterson. 2003. Modeling current and future potential wintering distributions of eastern North American monarch butterflies. P. Natl. Acad. Sci. USA. 100: 14063-14068.

Pleasants, J. M. and K. S. Oberhauser. 2012. Milkweed loss in agricultural fields because of herbicide loss: effect on the monarch butterfly population. Insect Conserv. Diver. 6: 135133.

Rall, B. C., O. Vucic-Pestic, R. B. Ehnes, M. Emmerson, and U. Brose. 2010. Temperature, predator-prey interaction strength and population stability. Glob. Change Biol. 16:21452157.

Reppert, S. M., R. J. Gegear, and C. Merlin. 2010. Navigational mechanisms of migrating monarch butterflies. Trends Neurosci. 33: 399-406.

Seabold, S. and Perktold, J.. 2010. Statsmodels: econometric and statistical modeling with Python, pp 57-61. In Proceedings, 9th Python in Science Conference, June 28 - July 3, Austin, TX. 
Sáenz-Romero, C., G. E. Rehfeldt, P. Duval, and R. A. Lindig-Cisneros. 2012. Abies religiosa habitat prediction in climatic change scenarios and implications for monarch butterfly conservation in Mexico. Forest Ecol. Manag. 275: 98-106.

Veteli, T. O., K. Kuokkanen, R. Julkunen-Tiitto, H. Roininen, and J. Tahvanainen. 2002. Effects of elevated $\mathrm{CO}_{2}$ and temperature on plant growth and herbivore defensive chemistry. Global Change Biol. 8: 1240-1252.

York, H. A. and K. S. Oberhauser. 2002. Effects of duration and timing of heat stress on monarch butterfly (Danaus plexippus) (Lepidoptera: Nymphalidae) development. J. Kansas Entomol. Soc. 75: 290-298.

Zalucki, M. P. 1982. Temperature and rate of development in Danaus plexippus L. and D chrysippus L. (Lepidoptera: Nymphalidae). J. Aust. Entomol. Soc. 21: 241-246.

Zipkin, E. F., L. Ries, R. Reeves, J. Regetz, and K. S. Oberhauser. 2012. Tracking climate impacts on the migratory monarch butterfly. Global Change Biol. 18: 3039-3049.

Zvereva, E. L. and M. V. Kozlov. 2006. Consequences of simultaneous elevation of carbon dioxide and temperature for plant-herbivore interactions: a metaanalysis. Global Change Biol. 12: 27-41.

van der Walt, S., S. C. Colbert, and G. Varoquaux. 2011. The NumPy Array: a structure for efficient numerical computation. Comput. Sci. Eng. 13: 22-30. 
Figure 1 (on next page)

Effects of temperate on monarch development time.

Monarch development time decreased as temperature increased in both Ambient (open circles) and Warmed (filled circles) plots. However, the effect of temperature on monarch larval development was stronger in Warmed plots. 
